\title{
EMBEDDED-ULTRASONICS STRUCTURAL RADAR FOR NONDESTRUCTIVE EVALUATION OF THIN-WALL STRUCTURES
}

\author{
Victor Giurgiutiu \\ Mechanical Engineering Department, University of South Carolina \\ Columbia, SC 29208, 803-777-8018, victorg@sc.edu \\ Jingjing Bao \\ Mechanical Engineering Department, University of South Carolina \\ Columbia, SC 29208, 803-777-1535; jbao0000@engr.sc.edu
}

\begin{abstract}
Embedded-Ultrasonics Structural Radar (EUSR) is a new concept and methodology for in-situ nondestructive evaluation (NDE) of thinwall structures. EUSR utilizes (a) a Piezo Wafer Active Sensors (PWAS) array embedded onto the structure; and (b) electronic modules for signal transmission/reception, processing, and interpretation. The EUSR methodology is developed as an extension of our previous work on the Lamb wave propagation NDE. Based on the study of the Lamb wave characteristic, the excitation signal is optimized to obtain good signal to noise ratio. Lamb wave speedfrequency curve is plotted to obtain the wave speed of the excitation signal. This wave speed is then used to map the EUSR data from time domain to distance domain, thus the locations of the reflectors can be visually displayed. The EUSR algorithm is adopted from the beamforming process currently used in phased-array radar applications. Each element in the PWAS array plays the role of both transmitter and receiver. A circuit is designed to change the role of the PWAS in a round robin fashion. The responses of the structure to all the excitation signals are collected. By applying the EUSR algorithm, an appropriate delay is applied to each signal in the data set to make them all focus on a direction denoted by angle $\phi$. When this angle $\phi$ is changed from 0 to 180 degrees, a virtual scanning beam is formed and a large area of the structure can be interrogated. The EUSR algorithm is explained in this paper. Experimental results from a proof-ofconcept EUSR system are also presented.

Keywords: in-situ diagnostics, phased-array, health monitoring, piezoelectric, ultrasonic, Lamb wave, piezoelectrics
\end{abstract}

\section{INTRODUCTION}

The use of ultrasonic techniques for damage detection, nondestructive inspection (NDI), nondestructive testing (NDT), and nondestructive evaluation (NDE) is well establishes (Krautkramer and Krautkramer, 1990; Blitz and Simpson; 1996; Rose; 1999)[1-3]. Two basic techniques are being generally used: (a) pulse-echo; and (b) pitch-catch. The pulse-echo method uses one transducer to send a short signal (pulse) and to detect the echoes of this signal generated by the backscatter of the signal from various material defects. The pulseecho method closely resembles the method used in finding a target using radar. Demirli and Saniie (2001) [4] used a model-based study of the patterns of ultrasonic backscatter echoes to extract information regarding the geometric shape, size, and orientation as well as microstructure of the examined object. The pitch-catch method uses two transducers, one to transmit, the other to receive the signal. A development of the pitch-catch method is found under the name of "acousto-ultrasonics" (Duke, 1988) [5]. Using this approach, Ravenscroft et al. (1991) [6] studied the diffraction of ultrasound by cracks and compared theoretical and experimental results with the pitch-catch method and time of flight technique. They found that, at certain angles, the reception of the scattered waves reaches a minimum, while other angles yield a maximum signal.

Ultrasonic transducers operated almost exclusively based on piezoelectric effect (Krautkramer and Krautkramer, 1990) [1]. Their structure, in general, consists of a piezoelectric oscillator disk, a protective layer and the damping block. Their action is normal to the contact surface. To generate obliquely incident wave, a wedgetransducer is needed. Due to the complexity of their construction, traditional ultrasonic transducers are bulky. In addition, to generate and receive the ultrasonic waves, special designed instrumentations are required. The advent of commercially available low-cost piezoceramics wafers has opened new opportunities for ultrasonic testing. Through their intrinsic electro-mechanical (E/M) coupling, the piezoceramics wafers can act as both sensors and actuators. Giurgiutiu and Zagrai (2000) [7] studied the properties of piezoelectric-wafer active sensors and coined the name PWAS.

The advantages of using a phased-array set of transducers in ultrasonic testing are multiple (Krautkramer and Krautkramer, 1990; Rose; 1999) [1,3]. The principles of phased-array ultrasonic inspection resemble the phased-array principles used in other applications (radar, sonar, etc.) Existing commercial phased array ultrasonic transducers are designed for detecting inhomogeneity in the specimen. They cannot be used for detecting failures on thin wall structures. Embedded-Ultrasonics Structural Radar (EUSR) is designed for in-situ NDE of thin-wall structures. EUSR consists of: (a) a PWAS array embedded onto the structure; and (b) electronic modules for signal 
transmission/reception, processing, and interpretation. The EUSR concept is introduced in this paper, brief theoretical description and some experimental results are also presented.

\section{LAMB WAVE PROPAGATION NDE}

Lamb waves are guided waves that exist in thin wall structures with free boundaries. For a plate having a thickness of the order of a wavelength or so, surface Rayleigh waves degenerate into Lamb waves (Viktorov, 1967) [8]. Lamb waves can propagate in infinite number of modes, either symmetrical or anti-symmetrical. The wave velocity depends on the product of frequency and material thickness. Investigations on Lamb and leaky Lamb waves have been carried out continuously since their discovery. They have been used in ultrasonic testing, especially for thin wall structures.

\section{Lamb Wave Speed}

Wave speed is one the most important parameters for ultrasonic testing. The Lamb wave speed is obtained by solving the Rayleigh-

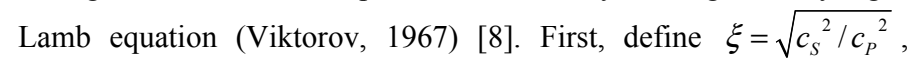
$\zeta=\sqrt{c_{S}{ }^{2} / c_{L}{ }^{2}}$, and $\bar{d}=k_{S} d$; where $c_{L}$ is the Lamb wave speed, and $d$ is the half thickness of the plate. In addition, also define Lamb wave

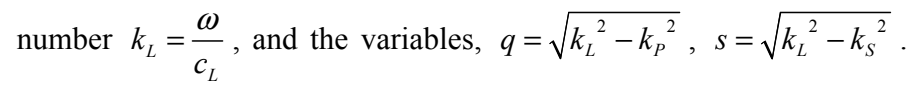

For symmetrical motion (Figure 1a), the Rayleigh-Lamb frequency equation can be written as

$$
\frac{\tan \left(\sqrt{1-\zeta^{2}} \bar{d}\right)}{\tan \sqrt{\xi^{2}-\zeta^{2}}}+\frac{4 \zeta^{2} \sqrt{1-\zeta^{2}} \sqrt{\xi^{2}-\zeta^{2}}}{\left(2 \zeta^{2}-1\right)^{2}}=0
$$

Then one can write the two components of the displacement as: (Viktorov, 1967) [8].

$$
\begin{aligned}
& U(x, z, t)=\operatorname{Re}\left[A k_{L}\left(\frac{\cosh (q z)}{\sinh (q d)}-\frac{2 q s}{k_{L}^{2}+s^{2}} \frac{\cosh (s z)}{\sinh (s d)}\right) e^{i\left(k_{L} x-\omega t \frac{\pi}{2}\right)}\right] \\
& W(x, z, t)=\operatorname{Re}\left[A q\left(\frac{\sinh (q z)}{\sinh (q d)}-\frac{2 k_{L}^{2}}{k_{L}^{2}+s^{2}} \frac{\sinh (s z)}{\sinh (s d)}\right) e^{i\left(k_{L} x-\omega t\right)}\right]
\end{aligned}
$$

For anti-symmetric motion (Figure 1b), the Rayleigh-Lamb frequency equation is

$$
\frac{\tan \left(\sqrt{1-\zeta^{2}} \bar{d}\right)}{\tan \sqrt{\xi^{2}-\zeta^{2}}}+\frac{\left(2 \zeta^{2}-1\right)^{2}}{4 \zeta^{2} \sqrt{1-\zeta^{2}} \sqrt{\xi^{2}-\zeta^{2}}}=0 .
$$

The two component of the displacement can be expressed as

$$
\begin{aligned}
& U(x, z, t)=\operatorname{Re}\left[A k_{L}\left(\frac{\cosh (q z)}{\cosh (q d)}-\frac{2 q s}{k_{L}^{2}+s^{2}} \frac{\cosh (s z)}{\cosh (s d)}\right) e^{i\left(k_{L} x-\omega t \frac{\pi}{2}\right)}\right] \\
& W(x, z, t)=\operatorname{Re}\left[A q\left(\frac{\sinh (q z)}{\cosh (q d)}-\frac{2 k_{L}^{2}}{k_{L}^{2}+s^{2}} \frac{\sinh (s z)}{\cosh (s d)}\right) e^{i\left(k_{L} x-\omega t\right)}\right]
\end{aligned}
$$
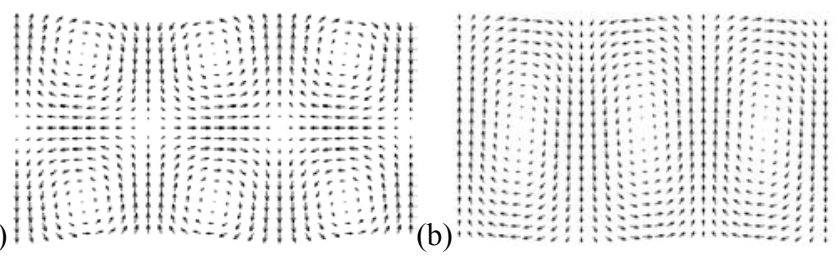

Figure 1 - Simulation of Lamb wave particle motion: (a) $S_{0}$ symmetric mode; (b) $A_{0}$ anti-symmetric mode
Figure $2 \mathrm{a}$ presents the dispersive (frequency dependant) symmetric and anti-symmetric $\left(\mathrm{S}_{0}\right.$ and $\left.\mathrm{A}_{0}\right)$ Lamb wave speeds in aluminum plates. Also presented in Figure 2 is the dispersive speed for the conventional (Bernoulli-Euler) flexural waves. One notices that, at low frequencies, the conventional flexural wave and the $\mathrm{A}_{0}$ Lamb wave speeds tend to coincide. At high frequencies, the Lamb wave speeds reach a horizontal asymptote, while the flexural waves continue to increase.

The group velocity of Lamb waves is important when examining the traveling of Lamb wave packs, as in the experiments described later in this paper. To calculate the group velocity of Lamb waves, we used the formula (Rose, 1999) [3]:

$$
c_{g}=c^{2}\left[c-(f d) \frac{\mathrm{d} c}{\mathrm{~d}(f d)}\right]^{-1}
$$

where $c$ is the Lamb wave phase velocity. Figure $2 \mathrm{~b}$ shows a plot of the group velocities vs. frequency for thin-gage aluminum with thickness $2 d=1.6 \mathrm{~mm}$. It is appreciated that, at low frequencies ( $\mathrm{f}<$ $50 \mathrm{kHz}$ ) the conventional flexure waves and the antisymmetric Lamb waves $\left(\mathrm{A}_{0}\right)$ group velocities are practically indistinguishable. However, at high frequencies, the behavior of the conventional flexure waves departs considerably from that of the Lamb waves.

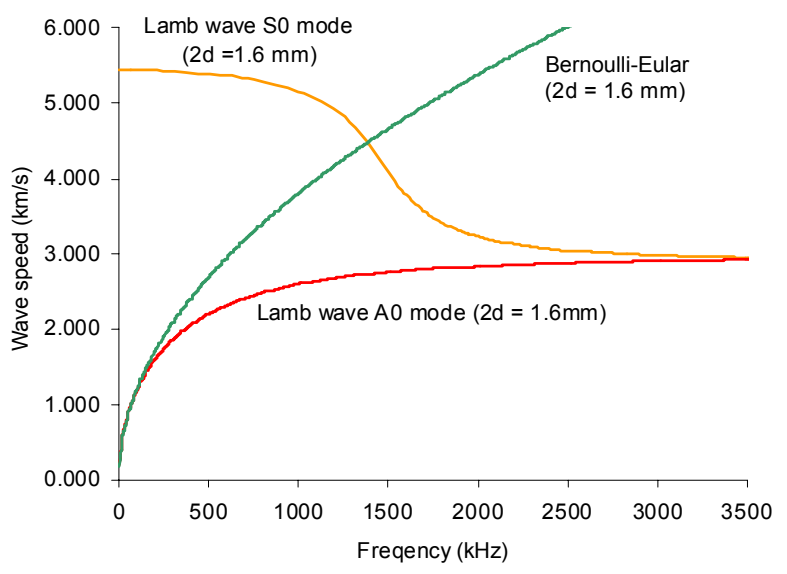

(a)

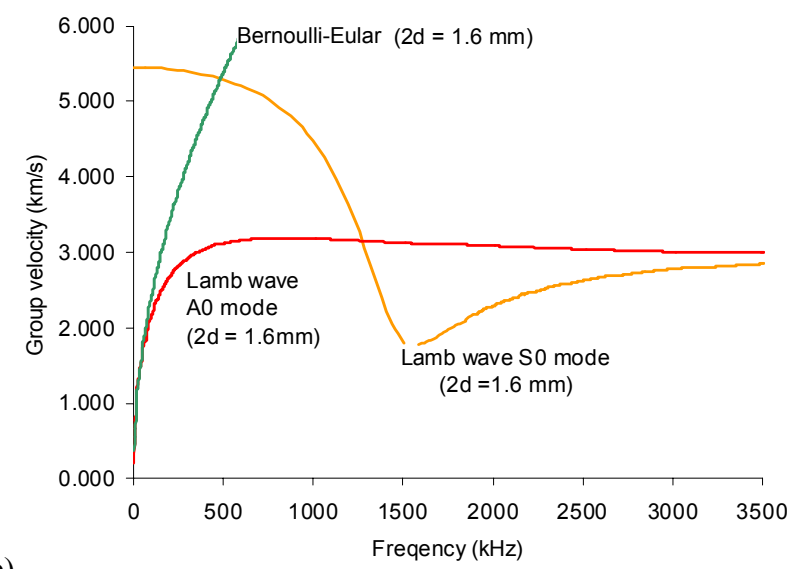

Figure 2 - Dispersion curves for classical flexure and Lamb waves in aluminum alloy structures: (a) phase velocity; (b) group velocity. 


\section{Lamb wave Experiments on Thin Plates}

Thin plate experiments were designed to measure the Lamb wave velocity. A $1.6 \mathrm{~mm}$ thick, 2024-aluminum alloy plate $(914 \mathrm{~mm} \mathrm{x}$ $504 \mathrm{~mm} \times 1.6 \mathrm{~mm}$ ) was instrumented with an array of eleven $7-\mathrm{mm} \times$ 7-mm PZT wafer active sensors. The sensor locations are given in Table 1. The sensors were connected with thin insulated wires to a 16channels signal bus and two 8-pin connectors (Figure 3). An HP33120A signal generator was used to generate a $300 \mathrm{kHz}$ windowed harmonic-burst excitation to active sensor \#11 with a $10 \mathrm{~Hz}$ repetition rate. Under the harmonic burst excitation, the active sensor generated a package of elastic waves that spread out into the entire plate according to a circular wave front pattern. A Tektronix TDS210 two-channel digital oscilloscope, synchronized with the signal generator, was used to collect the response signals from the active sensors. The two oscilloscope channels were digitally switched among the remaining 10 active sensors using a digitally controlled switching unit. A LabView data acquisition program was developed to control the signal switch and record the data from the digital oscilloscope. In addition, a Motorola MC68HC11 microcontroller was tested as an embedded stand-alone controlling option.

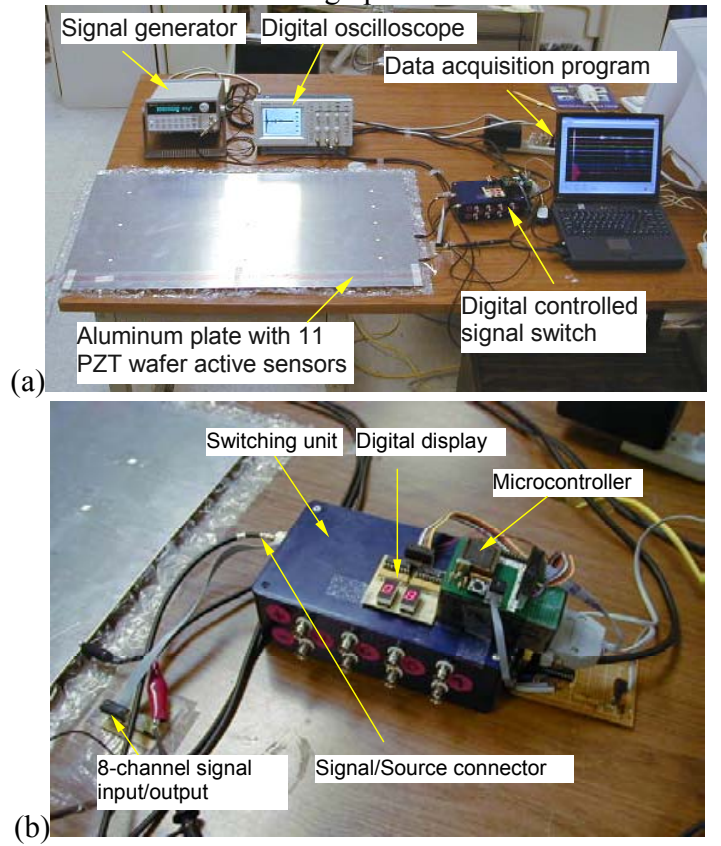

Figure 3 - Experimental setup for rectangular plate wave propagation experiment: (a) overall view showing the plate, active sensors, and instrumentation; (b) detail of the microcontroller and switch box.

The results of this investigation are shown in Figure 4. The first row shows the excitation signal, and a number of wave packages received in the pulse-echo mode. The first wave package corresponds to the wave rcveived from the transmitter PWAS, while the other wave packages are reflection from the plate edges. The time-of-flight (TOF) is consistent with the distance traveled by the wave. For the first wave packs, their TOF position is consistent with the distance between the transmitting and receiving PWAS. Figure 4(b) shows the correlation between TOF and distance. The consistency of the wave patterns is remarkable. These raw signals were processed using a narrow-band signal correlation algorithm followed by an envelope detection method. As a result, the exact TOF for each wave package could be precisely identified.
Table 1 Sensor location and time of arrival at $300 \mathrm{kHz}$

\begin{tabular}{|l|l|l|l|l|}
\hline$\#$ & $\mathrm{x}$ & $\mathrm{y}$ & $\mathrm{r}(\mathrm{mm})$ & $\mathrm{t}$ (micro-s) \\
\hline 1 & 100 & 100 & 761.6 & 147 \\
\hline 2 & 100 & 175 & 735.3 & 142 \\
\hline 3 & 100 & 250 & 715.9 & 138 \\
\hline 4 & 100 & 325 & 704.0 & 136 \\
\hline 5 & 100 & 400 & 700.0 & 135 \\
\hline 6 & 450 & 100 & 461.0 & 92 \\
\hline 7 & 450 & 250 & 380.8 & 77 \\
\hline 8 & 450 & 400 & 350.0 & 71 \\
\hline 9 & 800 & 100 & 300.0 & \\
\hline 10 & 800 & 250 & 150.0 & \\
\hline 11 & 800 & 400 & & \\
\hline
\end{tabular}

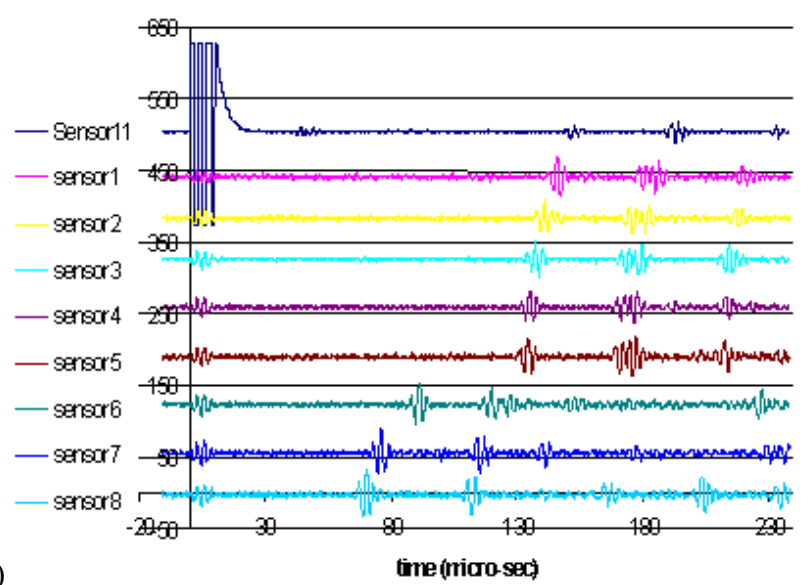

(a)

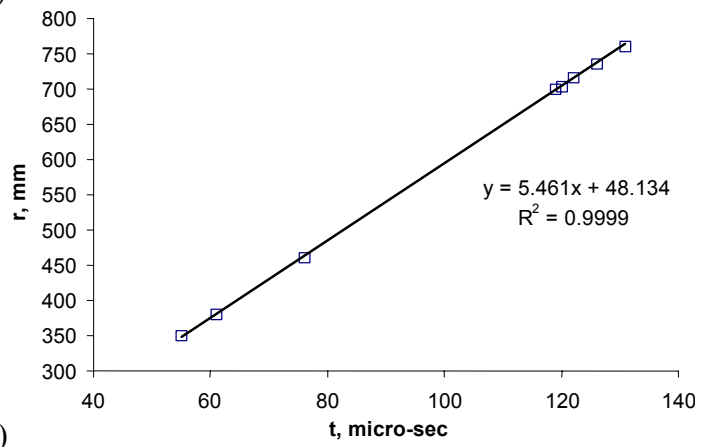

Figure 4 (a) Excitation signal and echo signals on active sensor 11, and reception signals on the other active sensor; (b) correlation between radial distance and time of flight. points:

These systematic experiments have proved several important

(1) The small non-intrusive PWAS used in these experiments were capable of exciting omnidirectional high-frequency Lamb waves in an aircraft-grade metallic plate.

(2) (2) The generated waves were of remarkable clarity and showed a $99.99 \%$ distance-TOF correlation that permitted accurate wave speed determination.

(3) (3) Pulse-echo methodology was successfully verified 


\section{Frequency Tuning Effects}

During our experiments, we investigated the effect of excitation frequency on the excited wave amplitude. It was found that, at low frequencies (e.g., $10 \mathrm{kHz}$ ) the excitation of flexural wave was much stronger than that of axial waves. However, as frequency increases beyond $150 \mathrm{kHz}$, the excitation of flexural waves decreases, while that of axial waves increases significantly. A "sweet spot" for axial wave excitation was found in the 300 to $400 \mathrm{kHz}$ range, as shown in Figure 5 . At $300 \mathrm{kHz}$, the first mixed wave pack and the axial pack peak. The second wave pack peaks at $350 \mathrm{kHz}$. Subsequently, for the plate experiments described in the next section, excitation at $300 \mathrm{kHz}$ was adopted as standard.

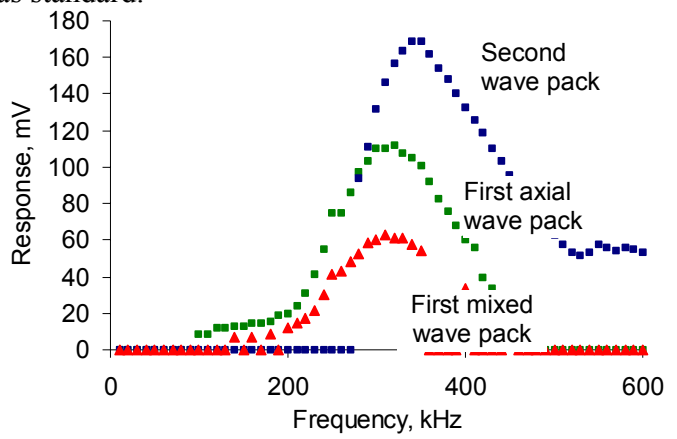

Figure 5 - Frequency tuning studies identified a maximum wave response around $300 \mathrm{kHz}$.

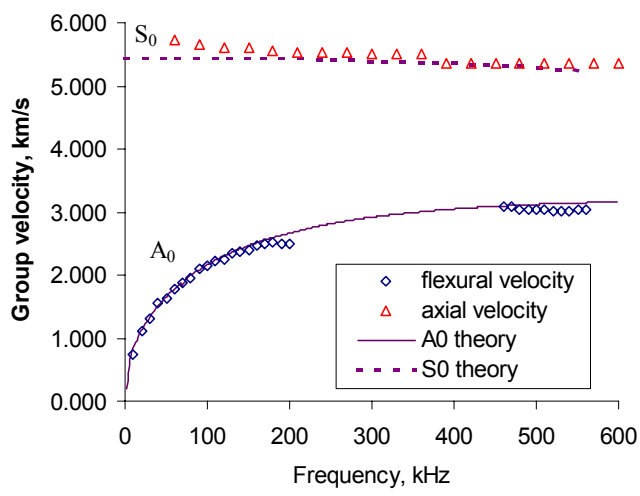

Figure 6 - Group velocity dispersion curves for Lamb-wave $A_{0}$ and $\mathrm{S}_{0}$ modes, theory vs. experiment.

\section{Experimental Determination of Group Velocity Dispersion $\underline{\text { Curves }}$}

Figure 6 shows a plot of the group velocity vs. frequency, resulted from our experimental measurements. Superposed on the same chart are the $A_{0}$ and $S_{0}$ group velocities curves predicted by Lamb wave theory. The concordance between the theoretical $\mathrm{A}_{0}$ group velocity and the measured $\mathrm{A}_{0}$ group velocity is remarkably good. The $\mathrm{A}_{0}$ data for the interval $200 \mathrm{kHz}$ to $450 \mathrm{kHz}$ was not measured because, in this interval, the flexural wave could not be excited due to the axial wave's dominance mentioned in the previous paragraph (cf. Figure 5). The $\mathrm{S}_{0}$ data also shows remarkably good concordance with the theoretical predictions, except at low frequencies $(f<100 \mathrm{kHz})$ where the excitation of axial waves is more difficult due to the flexural wave's dominance. Overall, the data presented in Figure 6 indicates that the theoretically predicted Lamb wave group velocities were experimentally confirmed.

\section{The Importance of High Frequency Excitation}

Our wave propagation simulation efforts were performed for a variety of frequencies in the range 10 to $600 \mathrm{kHz}$. However, low frequency waves are inappropriate for ultrasonic applications. To achieve damage detection with the pulse-echo method, the timewise length of the wave pack must be much less than the time taken for the echo to return. We observed that, at low frequencies (e.g., $10 \mathrm{kHz}$ ), the echo signal starts to appear before the incident signal has finished developing, thus markedly impeding the damage detection process. Hence, high excitation frequencies are needed. As the frequency increases, the wavelength decreases. This was especially apparent for flexural waves, which, at a given frequency, have a shorter wavelength than axial waves. With frequency of $300 \mathrm{kHz}$ we were able to successfully simulate both axial and flexural wave patterns, and to clearly identify defect-generated echo's as close as $30 \mathrm{~mm}$ from the source. For the detection of defects closer than $30 \mathrm{~mm}$, higher frequencies are required.

(a)

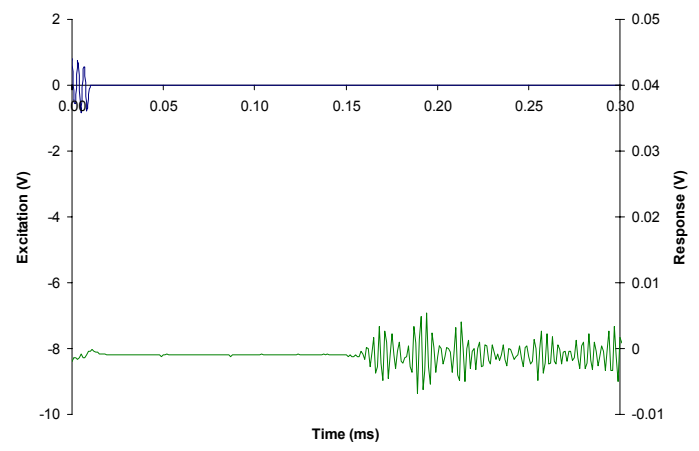

(b)

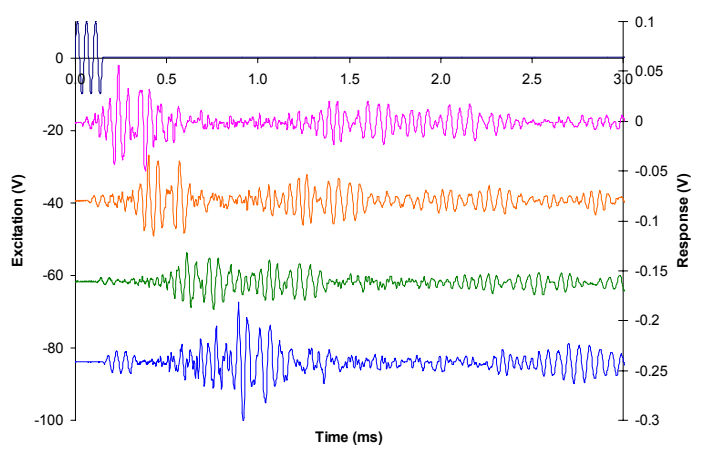

Figure 7 - Illustration of time resolution concept: (a) adequate time resolution at $300 \mathrm{kHz}$; (b) inadequate time resolution at 20 $\mathrm{kHz}$

\section{EMBEDDED-ULTRASONIC STRUCTURAL RADAR (EUSR) ALGORITHM}

The principle of operation of the embedded ultrasonic structural radar (EUSR) is derived, from two general principles (1) The principle of guided Lamb wave generation with piezoelectric wafer active sensors (PWAS); (2) The principles of conventional phasedarray radar

The guided Lamb waves generated by PWAS have the important property that they stay confined inside the walls of a thin-wall structure, and hence can travel over large distances. In addition, the 
guided wave can also travel inside curved walls, which makes them ideal for applications in the ultrasonic inspection of aircraft, missiles, pressure vessel, oil tanks, pipelines, etc. Lamb waves can exist in a number of dispersive modes. However, through smoothed tone-burst excitation and frequency tuning, it is possible to confine the excitation to a particular Lamb wave mode, of carrier frequency $F_{c}$, wave speed $c$, and wave length $\lambda=c / F_{c}$. Hence, the smoothed tone-burst signal generated by one PWAS is of the form:

$$
s_{T}(t)=s_{0}(t) \cos 2 \pi F_{c} t, 0<t<t_{p}
$$

where $s_{0}(t)$ is a short-duration smoothing window that is applied to the carrier signal of frequency $F_{c}$ between 0 and $t_{p}$.

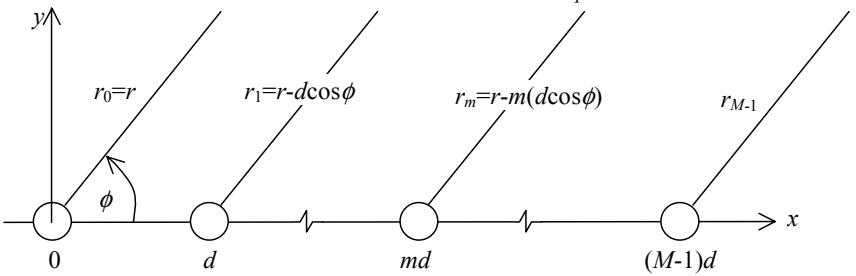

Figure 8 - Uniform linear array of $M$ omni-directional sensors (PWAS) spaced at pitch $d$

The principles of conventional phased-array radar applied to PWAS-generated guided waves assumes a uniform linear array of $M$ sensors (PWAS), with each PWAS acting as a pointwise omnidirectional transmitter and receiver. The PWAS in the array are spaced at the distance $d$, which is assumed much smaller than the distance $r$ to a generic, far-distance point, P. Since $d<<r$, the rays joining the sensors with the point $\mathrm{P}$ can be assimilated with a parallel fascicle, of azimuth $\phi$ (Figure 8 ).
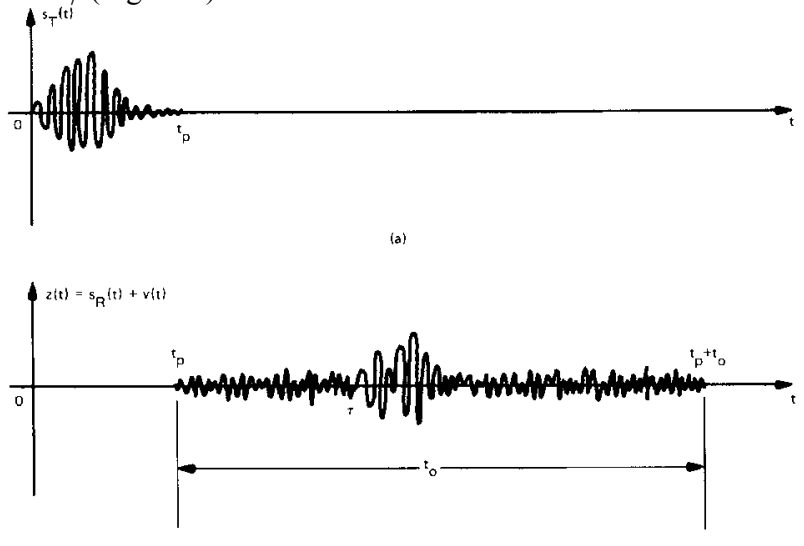

(b)

Figure 9 - The basis of pulse-echo method: (a) transmitted smooth-windowed tone-burst of duration $t_{p}$; (b) received signal to be analyzed for the duration $t_{0}$, starting at $t_{p}$, in order to identify the time of flight delay, $\tau$. (after Silvia, 1987) [9]

Because of the array spacing, the distance between one PWAS and the generic point $\mathrm{P}$ will be different from the distance between another PWAS and P. For the $m$-th PWAS, the distance will be shorted by $m(d \cos \phi)$. If all the PWAS are fired simultaneously, the signal from the $m$-th PWAS will arrive at P quicker by $\Delta_{m}(\phi)=m(d \cos \phi) / c$. If the PWAS are not fired simultaneously, but with some individual delays, $\delta_{m}, m=0,1, \ldots, M-1$, then the total signal received at point $\mathrm{P}$ will be:

$$
S_{\mathrm{P}}(t)=\frac{1}{r} \sum_{m=0}^{M-1} s_{\mathrm{T}}\left(t-\frac{r}{c}+\Delta_{m}(\phi)-\delta_{m}\right)
$$

where $1 / r$ represent the decrease in the wave amplitude due to the omni-directional 2-D radiation, and $r / c$ is the delay due to the travel distance between the reference PWAS $(m=0)$ and the point P. (waveenergy conservation, i.e., no dissipation, is assumed.)

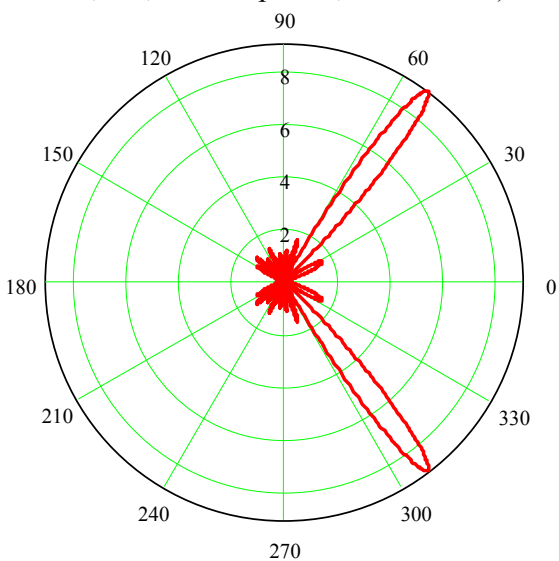

Figure 10 - Predicted beamforming pattern for a 9-sensor array (spacing $I=\lambda / 2$ ) with 53-deg target azimuth

\section{$\underline{\text { Transmitter beamforming }}$}

Beamforming at angle $\phi_{0}$ with an array of $M$ omni-directional sensors is based on the principles of constructive interference in the fascicle of parallel rays emanating from the array. The simplest way of achieving constructive interferences, is to have $\delta_{m}=m \Delta(\phi)$, such that Equation (9) becomes

$$
s_{\mathrm{P}}(t)=M \cdot \frac{1}{r} s_{\mathrm{T}}\left(t-\frac{r}{c}\right)
$$

i.e. an $M$ times increase in the signal strength with respect to a simple sensor. This leads directly to the beamforming principle, i.e., if $\delta_{m}=m \frac{d}{c} \cos \left(\phi_{0}\right)$, and since $\Delta_{m}=m \frac{d}{c} \cos (\phi)$, then constructive interference (beamforming) takes place when $\cos (\phi)=\cos \left(\phi_{0}\right)$, i.e. at angles $\phi=\phi_{0}$ and $\phi=-\phi_{0}$. Thus, the forming of a beam at angles $\phi_{0}$ and $-\phi_{0}$ is achieved through delays in the firing of the sensors in the array. Figure 10 shows the beam forming pattern for $\phi_{0}=53 \mathrm{deg}$.

\section{Receiver beamforming}

The receiver beamforming principles are reciprocal to those of the transmitter beamforming. If the point $\mathrm{P}$ is an omni-directional source at azimuth $\phi_{0}$, then the signals received at the $m$-th sensor will arrive quicker by $m \Delta_{0}(\phi)=m\left(d \cos \phi_{0}\right) / c$. Hence, we can synchronize the signals received at all the sensors by delaying them by $\delta_{m}\left(\phi_{0}\right)=m \frac{d}{c} \cos \left(\phi_{0}\right)$

\section{Phased-array pulse-echo}

Assume that a target exists at azimuth $\phi_{0}$ and distance $R$. The transmitter beamformer is sweeping the azimuth in increasing angles $\phi$ and receives an echo when $\phi=\phi_{0}$. The echo will be received on all sensors, but the signals will not be in synch. To synchronize the sensors signals, the delays $\delta_{m}\left(\phi_{0}\right)=m \frac{d}{c} \cos \left(\phi_{0}\right)$ need to be applied. The process is as follows. The signal send by the transmitter beamformer is an $M$ times boost or the original signal: 


$$
S_{\mathrm{P}}(t)=\frac{M}{R} S_{\mathrm{T}}\left(t-\frac{2 R}{c}\right)
$$

At the target, the signal is backscattered with a backscatter coefficient, $A$. Hence, the signal received at each sensor will be $\frac{A \cdot M}{R^{2}} s_{\mathrm{T}}\left(t-\frac{2 R}{c}+\Delta_{m}(\phi)\right)$. The receiver beamformer assembles the signals from all the sensors with the appropriate time delays, i.e.,

$$
s_{\mathrm{R}}(t)=\frac{A \cdot M}{R^{2}} \sum_{m=0}^{M-1} s_{\mathrm{T}}\left(t-\frac{2 R}{c}+\Delta_{m}(\phi)-\delta_{m}\right)
$$

Constructive interference between the received signals is achieved when $\delta_{m}=m \frac{d}{c} \cos \left(\phi_{0}\right)$. Thus, the assembled receive signal will be again boosted $M$ times, with respect to the individual sensors, i.e.,

$$
s_{\mathrm{R}}(t)=\frac{A \cdot M^{2}}{R^{2}} \sum_{m=0}^{M-1} s_{\mathrm{T}}\left(t-\frac{2 R}{c}\right)
$$

The time delay between the receive signal, $s_{\mathrm{R}}(t)$ and the transmit signal $s_{\mathrm{T}}(t)$, is

$$
\tau=\frac{2 R}{c}
$$

Measurement of the time delay $\tau$ observed in $s_{\mathrm{R}}(t)$ allows one to calculate the target range, $R=c \tau / 2$.

Table $2 M \times M$ matrix of signal primitives generated in a round-robin phase-array activation of the active-sensor array

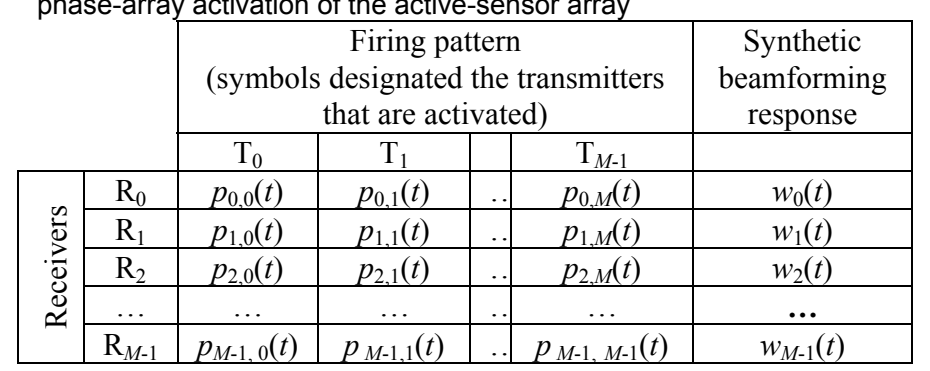

\section{Practical Implementation}

The signal generation and collection proceeds as follows: In a round-robin fashion, one active sensor at a time is activated as transmitter. The reflected signals are received at all the sensors: the activated sensor acts in pulse-echo mode, i.e. as both transmitter and receiver, the other sensors act as passive sensors. Thus an $M \times M$ matrix of signal primitives is generated (Table 1).

The signal primitives are assembled into synthetic beamforming responses using the synthetic beamformer algorithm equation (10). The delays, $\delta_{j}$, are selected in such a way as to steer the interrogation beam at a certain angle, $\phi_{0}$. The synthetic-beam sensor responses, $w_{i}(t)$, synthesized for a transmitter beam with angle $\phi_{0}$, are assembled by the receiver beamformer into the total received signal, $s_{R}(t)$, using the same delay as for the transmitter beamformer. However, this method presumes that the target angle $\phi_{0}$ is know a priori. In general applications, the target angle is not know, and needs to be determined. Hence, we write the received signal as a function of the parameter $\phi_{0}$, using the array unit delay for the direction $\phi_{0}$ as $\Delta_{0}\left(\phi_{0}\right)=\frac{d}{c} \cos \phi_{0}$. In practical implementation of the method, we have used a spline interpolation approach to implement the time shifts with accuracy at times that fall in between the fixed values of the sampled time.
A coarse estimate of the target direction is obtained by using an azimuth sweep technique, in which the beam angle, $\phi_{0}$, is modified until the maximum received energy is obtained, i.e.,

$$
\max E_{\mathrm{R}}\left(\phi_{0}\right), \quad E_{\mathrm{R}}\left(\phi_{0}\right)=\int_{t_{p}}^{t_{p}+t_{0}}\left|s_{\mathrm{R}}\left(t, \phi_{0}\right)\right|^{2} d t
$$

After a coarse estimate of the target direction is found $\phi_{0}$, the actual round-trip time of flight, $\tau_{\mathrm{TOF}}$, is calculated using an optimal estimator, e.g., the cross-correlation between the received and the transmitted signal:

$$
y(\tau)=\int_{t_{p}}^{t_{p}+t_{0}} s_{\mathrm{R}}(t) s_{\mathrm{T}}(t-\tau) d t
$$

Then, the estimated $\tau_{\mathrm{TOF}}=2 R / c$ is attained at the value of $\tau$ where $y(\tau)$ is maximum. Hence, the estimated target distance is

$$
R_{\text {exp }}=c \tau_{\mathrm{TOF}} / 2
$$

This algorithm works best for targets in the far field, for which the "parallel-rays" assumption holds.

For targets in the near and intermediate field, a more sophisticated self-focusing algorithm, that uses triangulation principles, is used. This algorithm is an outgrowth of passive-sensors target-localization methodologies (Silvia, 1987[9].) The self-focusing algorithm modifies the delay times for each synthetic-beam response, $w_{i}(t)$, such that it maximizes the total response, by finding the focal point of individual responses, i.e., the common location of the defect that generated the echoes recorded at each sensor. For very close range targets, SAFT techniques (Shandiz and Gaydecki; 1999) are utilized.

\section{EUSR SYSTEM DESIGN}

The EUSR system consists of three major modules: the PWAS array, the DAQ module, and the Signal Processing module. A system diagram is shown in Figure 11.

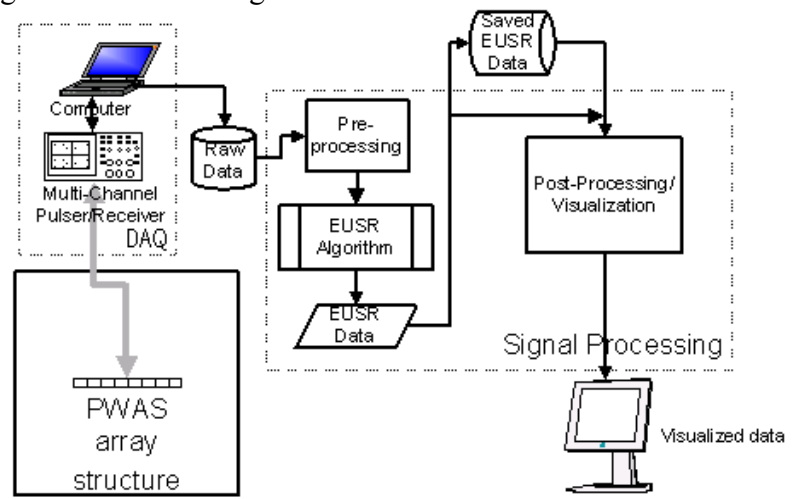

Figure 11 - data flow diagram of the EUSR LabVIEW program

A proof-of-concept system was built in Laboratory for Active Materials and Smart Structures (LAMSS) in University of South Carolina to evaluate the feasibility and capability of the EUSR system. Three specimens were used in the experiments. These specimens were 1220-mm (4-ft) square panel of 1-mm (0.040-in) thick 2024-T3 Alclad aircraft grade sheet metal stock. One of the specimens was pristine, and was used to obtain baseline data. The other two were manufactured with simulated cracks. The size of the cracks were 19 $\mathrm{mm}(3 / 4-\mathrm{in})$ long, $0.127 \mathrm{~mm}(0.005$-in) wide, and were placed: (a) broadside at $R=305 \mathrm{~mm}$ from the PWAS array; and (b) offside at $R=$ $453 \mathrm{~mm}, \phi_{0}=130.3 \mathrm{deg}$. The EUSR system were used to detect the locations of the cracks. 
PWAS array is an array of small size, inexpensive, and nonintrusive piezoelectric-wafer active sensors. The transducers are made from American Piezo Ceramic Inc. APC850 piezo ceramic wafers, $7 \mathrm{~mm} \times 7 \mathrm{~mm}$ in size. These transducers can work as both transmitters and receivers. The EUSR system tested in the LAMSS used an array consists of 9 PWAS placed on a straight line and spaced at distance $l=$ $\lambda / 2$, where $\lambda$ is the wavelength of the guided wave propagating in the thin-wall structure. According to elementary wave mechanics, $\lambda=c / f$, where $c$ is the wave speed and $f$ is the wave frequency. Our proof-ofconcept experiments, performed on 1-mm gauge 2024-T3 aluminum alloy aircraft-grade sheet material, identified the first optimum excitation frequency at $300 \mathrm{kHz}\left(\mathrm{S}_{0}\right.$ mode) with the corresponding wave speed $c=5.440 \mathrm{~km} / \mathrm{s}$, and wavelength $\lambda=18 \mathrm{~mm}$. Hence, the PWAS spacing for our proof-of-concept PWAS array was selected $d=$ $9 \mathrm{~mm}$ (Figure 12).

DAQ module uses a computer to control and collect data from a multi-channel pulser/receiver. The multi-channel pulser/receiver consists of (a) a HP33120A arbitrary signal generator, (b) a Tektronix TDS210 digital oscilloscope, and (c) a digital controlled signal switching unit. The HP33120A arbitrary signal generator was used to generate a $300 \mathrm{kHz}$ Hanning windowed tone-burst excitation with a 10 $\mathrm{Hz}$ repetition rate. Under the Hanning windowed tone-burst excitation, one element in the PWAS array generates a package of elastic waves that spread out into the entire plate with a circular wave front (omnidirectional pattern.) The Tektronix TDS210 digital oscilloscope, synchronized with the signal generator, was used to collect the response signals from the PWAS array. One of the oscilloscope channels was switched among the remaining elements in the PWAS array by using a digitally controlled switching unit. A LabVIEW computer program was developed to control the digital signal switching unit and record the data from the digital oscilloscope to a group of raw data files.

(a).

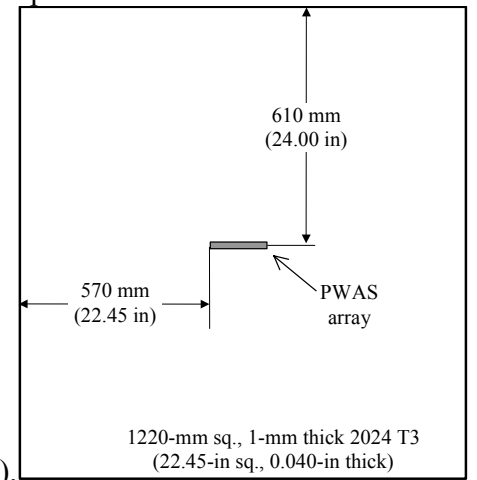

Figure 12 - Proof-of-concept EUSR construction: (a) 4-ft square plate with 9-element PWAS array at its center; (b) details of the 9-element PWAS array with 7-mm square PWAS;

The signal-processing module reads the raw data files and process these data with EUSR algorithm. Although the EUSR algorithm is not computationally intensive, the large amount of data points in each signal made this step time consuming. Hence the resulting data is saved in a EUSR data file on computer for later retrieval. This data file also enables other programs to access the EUSR data.

After being processed, the data was transformed from time domain to 2-D plane domain. Based on the EUSR algorithm, the resulting data file is a collection of signals that represent the structure response at different angles, defined by the parameter $\phi$. In nother words, they represent the response when EUSR scanning beam turned onto angle $\phi$.

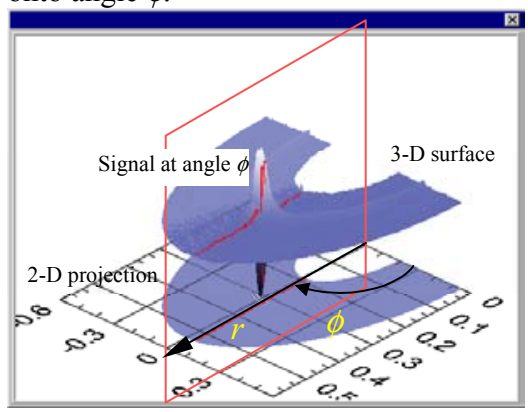

Figure 13 - Data visualization of the EUSR results.

Knowing the Lamb wave speed $c$, by using $r=c t$, this signal can be transformed from voltage $V$ vs. time $t$ to voltage $V$ vs. distance $r$. The signal detected at angle $\phi$ an be plotted on a 2-D plane at angle $\phi$. Since angle $\phi$ was stepped from 0 to $180^{\circ}$, at constant increments, the plots covered a half space. These plots generates a 3-D surface, which is a direct mapping of the structure being interrogated, with the $z$ value of the 3-D surface representing the response voltage from that $(x, y)$ location. Recall that the voltage is related to the reflection amplitude level, so this surface also represents the intensity of the reflections. If we present the $z$ value in color scale, then the 3-D surface is project to the 2-D plane, and the color of each point on the plane represents the intensity of the reflections.

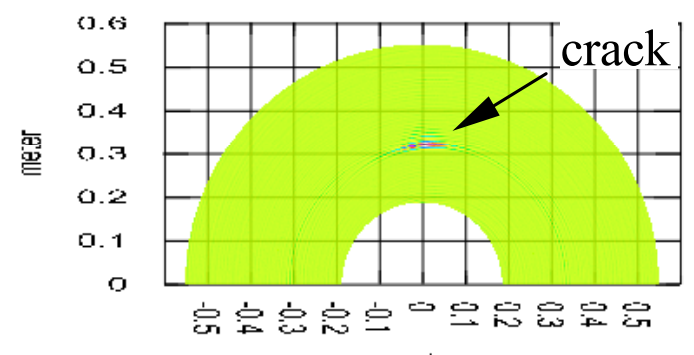

(a)

rmeler

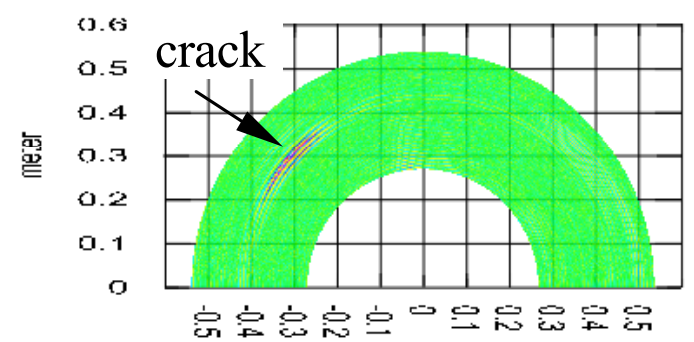

(b)

meler

Figure 14 - Visualized EUSR results. The data are from specimens with crack at (a) broadside ( $\left.\phi_{0}=90 \mathrm{deg}\right)$ and (b) offside $\left(\phi_{0}=136 \mathrm{deg}\right)$.

The two pictures in Figure 14 show the EUSR results from the proof-of-concept system. The grids represent one half of the 4-ft square specimen plate. The shaded area is a projection of the 3-D surface; the amplitude is presented in color/grayscale intensity.

Figure 14 (a) is from the broadside specimen. The small area with darker color represents high amplitude echo (reflected wave) generated when the scanning angular beam intercepted the crack. From the picture, this area is located at a distance of 0.31 meters from the center of the plate and at 90 degrees. Recall that the simulated 
crack on this specimen is $305 \mathrm{~mm}$ (0.305 meters) from the PWAS array, and at $\phi_{0}=90$ degrees. The dark area in the EUSR result predicted the simulated crack perfectly.

Similarly, Figure 14 (b) is from the offside specimen, and the darker area predicted the simulated crack at a location $0.3 \sqrt{2}$ meters from the center, 135 degrees, which estimated the real location of the simulated crack perfectly.

\section{CONCLUSION}

The main contribution of this paper lies in clarifying, through theoretical simulation and experimental measurements, the major aspects associated with using Piezoelectric-Wafer Active Sensors (PWAS) in conjunction with Embedded Ultrasonics Structural Radar (EUSR) for damage detection and in-situ structural health monitoring. The systematic theoretical and experimental investigations presented here have proved the following essential points:

1. The small $(7-\mathrm{mm} \times 7-\mathrm{mm} \times 0.2 \mathrm{~mm})$ non-intrusive piezoelectric-wafer active sensors (PWAS) used in these experiments were capable of exciting axial and flexural Lamb waves that propagate in an omnidirectional circular wave front throughout the thin-gage plate structures. It is conceivable that similar propagation will also happen in thin-gage shells typical of aerospace structures.

2. The same PWAS were also capable of capturing the elastic wave signals, thus proving their dual transmitter-receptor function. The captured signals were of remarkable strength (up to $50 \mathrm{mVpp}$ ) and could be used directly without amplification or other signal conditioning.

3. The pulse-echo technique, that is essential for ultrasonic damage detection was successfully demonstrated

4. The waves generated during this experiments showed remarkable clarity and could be easily interpreted. The experiments displayed a very good correlation between the time of flight and the wave path length that permitted the determination of the wave speed (group velocity) with up to 99.9\% accuracy. This high accuracy will ensure accurate detection of internal defect when the method is applied in structural health monitoring.

5. EUSR algorithm is suitable for PWAS array signal processing, and proved to be efficient for detecting crack in structure. With appropriately designed hardware and software, in-situ, real-time thin wall structure health monitoring through EUSR is feasible.

The results reported in this paper open the path for applying the well-established theories and methodologies of ultrasonic testing and the radar theory to this new emerging field of embedded ultrasonics. The use of small and inexpensive piezoelectric-wafer active sensors instead of the conventional costly and bulky ultrasonic transducers opens the opportunity for creating arrays of embedded ultrasonic active sensors that can non-intrusively perform in-situ structural health monitoring. Though the active-sensor experiments reported here were conducted only on thin metallic plates of simple geometries, the findings of this study can be easily extended to practical geometries and to composite material structures.

\section{ACKNOWLEDGMENTS}

The financial support of Department of Energy through the Sandia National Laboratories, contract doc. \# BF0133 is thankfully acknowledged. Sandia National Laboratories is a multi-program laboratory operated by Sandia Corporation, a Lockheed Martin
Company, for the United States Department of Energy under contract DE-AC04-94AL85000.

\section{REFERENCES}

1. Krautkramer, J.; Krautkramer, H. (1990) Ultrasonic Testing of Materials, Springer-Verlag, 1990.

2. Blitz, J.; Simpson, G. (1996) Ultrasonic Methods of NonDestructive Testing, Chapman \& Hall, 1996.

3. Rose, J. L. (1999) Ultrasonic Waves in Solid Media, Cambridge University Press, 1999.

4. Demirli, R.; Saniie, J. (2001) "Model-Based Estimation of Ultrasonic Echoes" IEEE Transactions of Ultrasonics, Ferroelectrics, and Frequency Control, Vol. 48, No. 3, May 2001, pp. 787-811

5. Duke, J. C. Jr. (1988) Acousto-Ultrasonics - Theory and Applications, Plenum Press, 1988.

6. Ravenscroft, F. A.; Newton, K.; Scruby, C. B. (1991) "Diffraction of Ultrasound by Cracks: Comparison of Experiment with Theory", Ultrasonics, Vol. 29, January 1991, pp. 29-37

7. Giurgiutiu, V., Zagrai, A. (2001) "Embedded Self-Sensing Piezoelectric Active Sensors for On-Line Structural Identification", Submitted to: Transactions of ASME, Journal of Vibration and Acoustics, January 2001.

8. Viktorov, I. A. (1967) Rayleigh and Lamb waves - Physical Theoryand Applications, Plenum Press, NY, 1967.

9. Silvia, M. T. (1987) “Time Delay Estimation”, in Handbook of Digital Signal Processing, D. F. Elliot (Ed.), Academic Press, 1987 